\title{
Is in vitro fertilization associated with preeclampsia? A propensity score matched study
}

\author{
Noriyoshi Watanabe ${ }^{1}$, Takeo Fujiwara2 ${ }^{2}$, Tomo Suzuki ${ }^{1}$, Seung Chik Jwa ${ }^{2}, K^{\prime}$ Kosuke Taniguchi ${ }^{1}$, Yuji Yamanobe ${ }^{3}$,
} Kazuto Kozuka ${ }^{3}$ and Haruhiko Sago ${ }^{1}$

\begin{abstract}
Background: Although an increased risk of preeclampsia in pregnancies conceived by in vitro fertilization (IVF) has been reported, it remains unknown whether IVF is associated with preeclampsia. In the present study, we sought to investigate whether IVF is associated with preeclampsia in pregnant women using propensity score matching analysis.

Methods: This study included 3,084 pregnant women who visited the National Center for Child Health and Development before 20 weeks of gestation without hypertension or renal disease and delivered a singleton after 22 weeks of gestation between 2009 and 2011. Of the 3084 patients, 474 (15.4\%) conceived by IVF (IVF group) and 2,610 (84.6\%) conceived without IVF (non-IVF group). The propensity score for receiving IVF was estimated using multiple logistic regression with 27 maternal and paternal variables. This model yielded a c-statistic of 0.852 , indicating a strong ability to differentiate between those conceiving with and without IVF. The association between IVF and onset of preeclampsia was assessed by the propensity matched sample (pair of $\mathrm{N}=474$ ).
\end{abstract}

Results: There were 46 preeclampsia cases (1.5\%) in the total study population, with a higher proportion of cases in the IVF group (15 cases, 3.2\%) than the non-IVF group (31 cases, 1.2\%). Before propensity score matching, the IVF group was 2.72 (95\% confidence intervals [CI]: 1.46-5.08) times more likely to have preeclampsia when unadjusted, and 2.32 (95\% Cl: 1.08-4.99) times more likely to have preeclampsia when adjusted for maternal and paternal variables by logistic regression. After propensity score matching, the IVF group did not show a significantly greater association with preeclampsia compared to the non-IVF group (odds ratio: 2.50, 95\% Cl: 0.49-12.89), although point estimates showed a positive direction.

Conclusions: Propensity score matching analysis revealed that the association between IVF and preeclampsia became weaker than when conventional adjustments are made in multivariate logistic regression analysis, suggesting that the association between IVF and preeclampsia might be confounded by residual unmeasured factors.

Keywords: Blood pressure, In vitro fertilization, Preeclampsia, Propensity score, Assisted reproductive technology

\section{Background}

Preeclampsia is a major obstetric problem worldwide that causes significant maternal and perinatal morbidity and mortality [1]. Several risk factors have been identified, including advanced maternal age, primiparity, obesity, chronic hypertension, renal disease, pre-gestational diabetes mellitus, and autoimmune disease [2,3]. In addition, several

\footnotetext{
* Correspondence: fujiwara-tk@ncchd.go.jp

2Department of Social Medicine, National Research Institute for Child Health and Development, 2-10-1 Okura, Setagaya, Tokyo 157-8535, Japan Full list of author information is available at the end of the article
}

studies, including a systematic review and a meta-analysis, have reported an increased risk of preeclampsia in pregnancies conceived by in vitro fertilization (IVF) [4-7]. However, these studies used limited adjustment for confounders, such as age or parity. Thus, it remains unknown whether IVF directly induces preeclampsia. A randomized control trial is the ideal study design for investigating the impact of IVF on preeclampsia, as it allows for adjusting for known and unknown confounders. However, randomized assignment of IVF treatment is impossible in studies of pregnant women.

\section{Biomed Central}


Propensity score matching analysis is a statistical tool which can minimize selection bias and confounding factors in observational studies [8]. Estimating a propensity score for IVF treatment and matching based on the score may help determine whether IVF has a causal effect on preeclampsia. To the best of our knowledge, no study has examined the association between IVF and the incidence of preeclampsia using this statistical tool. Thus, we aimed to investigate the association between IVF and the incidence of preeclampsia by using a propensity score matching model.

\section{Methods \\ Participants}

We collected data of women who delivered between June 2009 and June 2011 at the National Center for Child Health and Development (NCCHD) in Tokyo, Japan. This study was approved by the NCCHD ethics committee. All pregnant women were Japanese. Women who delivered singleton neonates at $\geq 22$ weeks of gestation without hypertension or renal disease were enrolled in the study. In total, we identified 3,084 women who met our study criteria (eligible study sample), including 474 women who received IVF (IVF group) and 2,610 women who did not (non-IVF group). All 3,084 study participants had only one pregnancy during this study period. Written informed consent was not obtained from participants because the study involved a secondary data analysis and such studies do not require consent according to the NCCHD ethics committee.

\section{Assessment of preeclampsia}

Preeclampsia was diagnosed after 20 weeks of gestation if the pregnant woman had a blood pressure (BP) $>140$ / $90 \mathrm{mmHg}$ with proteinuria (>300 mg/24 hours). Earlyonset preeclampsia was defined as preeclampsia diagnosed at $<32$ weeks of gestation, and late-onset preeclampsia was defined as preeclampsia diagnosed at $32+$ weeks of gestation. Severe preeclampsia was diagnosed as BP $>160 /$ $110 \mathrm{mmHg}$ with proteinuria (>2 g/24 hours). Women received routine prenatal checkups every four weeks up to the $28^{\text {th }}$ week of gestation, then every two weeks up to the $36^{\text {th }}$ week of gestation, and then weekly up to delivery. At each routine prenatal checkup, BP was measured in the sitting position with the right arm held at heart level after a five-minute rest period using an automated sphygmomanometer (Omron BP203RVIII oscillometer, Nippon Colin, Tokyo, Japan). Urine protein levels were assessed at each prenatal checkup by a dipstick test of a single voided urine sample. When the pregnant woman had an abnormal BP $(>140 / 90 \mathrm{mmHg})$ and urine test $(1+$ proteinuria on dipstick) at a routine prenatal checkup, she was hospitalized for further evaluation of $\mathrm{BP}$ and urine protein and for bed rest.

\section{Baseline characteristics and pregnancy outcomes}

Maternal characteristics, including age, age at marriage, parity, number of abortions, working status (full-time, part-time, or housewife), height, weight before pregnancy, smoking and drinking during pregnancy, family history of hypertension, mean systolic and diastolic BP before 20 weeks of gestation, and underlying diseases (central nervous system disease, cerebrovascular disease, asthma, respiratory disease other than asthma, gastrointestinal disease, liver disease, hematologic disease, cardiovascular disease, hyperthyroidism, hypothyroidism, psychiatric disease, autoimmune disease, and preexisting diabetes mellitus), were collected from the perinatal database and medical charts. Paternal factors, including age, age at marriage, height, weight, and smoking status, were also collected. Missing data for continuous variables (maternal age at marriage, 28 cases [0.9\%]; maternal height, 25 cases [0.8\%]; maternal weight, 26 cases [0.8\%]; paternal age at delivery, 219 cases [7.1\%]; paternal age at marriage, 239 cases [7.7\%]; paternal height, 35 [1.1\%]; and paternal weight, 35 [1.1\%]) were substituted with the average value of the variable. Missing data for categorical variables were treated as dummy variables (working status, 21 cases [0.7\%]; family history of hypertension, 235 cases [7.6\%]; paternal smoking, 65 cases $[2.1 \%])$.

\section{Statistical analysis}

Maternal and paternal characteristics were compared between the IVF and non-IVF groups using chi-squared or Fisher's exact tests for categorical variables and t-tests for continuous variables.

Because IVF procedures were not randomly assigned in this population, potential confounding and selection biases were accounted for by developing a propensity score for the IVF procedure. This propensity score was determined using a multivariate logistic regression model adjusting for maternal and paternal variables. These variables included those listed above as baseline characteristics, except for drinking during pregnancy and two types of underlying diseases (central nervous system disease and cerebrovascular disease), due to their low frequency $(<0.9 \%)$. Twenty-seven independent variables were thus used to determine the propensity score. This model yielded a c-statistic of 0.852 , indicating a strong ability to differentiate between those conceiving with and without IVF. The 474 IVF cases were then matched one-to-n with 474 non-IVF cases with the closest propensity score (nearest neighbor matching) $[9,10]$. The maximum difference in propensity score between each matched pair was 0.057. Baseline characteristics and pregnancy outcomes in the eligible study sample $(\mathrm{n}=3084)$ and propensity-matched study sample $(\mathrm{n}=948)$ are shown in Table 1. Further, characteristics of preeclampsia (prevalence, timing of onset, and severity), BP during mid- (28-32 weeks 
Table 1 Baseline characteristics of the eligible study sample and propensity score matched sample

\begin{tabular}{|c|c|c|c|c|c|c|c|}
\hline & & \multicolumn{3}{|c|}{$\begin{array}{l}\text { Eligible study sample } \\
\qquad(\mathrm{n}=3084)\end{array}$} & \multicolumn{3}{|c|}{$\begin{array}{l}\text { Propensity score matched sample } \\
\qquad(\mathrm{n}=948)\end{array}$} \\
\hline & & $\begin{array}{l}\text { IVF group } \\
(\mathrm{n}=474)\end{array}$ & $\begin{array}{l}\text { Non-IVF group } \\
(\mathrm{n}=2610)\end{array}$ & $P$ value & $\begin{array}{l}\text { IVF group } \\
(\mathrm{n}=474)\end{array}$ & $\begin{array}{l}\text { Non-IVF group } \\
(n=474)\end{array}$ & $P$ value \\
\hline \multicolumn{8}{|l|}{ Maternal demographics } \\
\hline Age & Year, mean (SD) & $38.3(3.3)$ & $34.8(4.2)$ & $<0.001$ & $38.3(3.3)$ & $38.4(3.3)$ & 0.97 \\
\hline Age at marriage & Year, mean (SD) & $30.8(4.1)$ & $29.3(3.8)$ & $<0.001$ & $30.8(4.1)$ & $31.0(4.1)$ & 0.91 \\
\hline \multirow[t]{2}{*}{ Parity } & $0, \mathrm{n}(\%)$ & $370(78.1)$ & $1319(50.5)$ & $<0.001$ & $370(78.1)$ & $378(79.8)$ & 0.74 \\
\hline & $\geq 1, n(\%)$ & $104(21.9)$ & $1291(49.5)$ & & $104(21.9)$ & $96(20.3)$ & \\
\hline \multirow[t]{3}{*}{ Abortion } & $0, \mathrm{n}(\%)$ & $291(61.4)$ & $1767(67.7)$ & 0.009 & $291(61.4)$ & $304(64.1)$ & 0.39 \\
\hline & $1-2, n(\%)$ & $152(32.1)$ & $734(28.1)$ & & $152(32.1)$ & $136(28.7)$ & \\
\hline & $3 \leq, n(\%)$ & $31(6.5)$ & $109(4.2)$ & & $31(6.5)$ & $34(7.2)$ & \\
\hline \multirow[t]{4}{*}{ Work } & Homemaker, n (\%) & $189(39.9)$ & $1122(43.0)$ & 0.31 & $189(39.9)$ & $190(40.1)$ & 0.93 \\
\hline & Full time, n (\%) & $248(52.3)$ & $1268(48.6)$ & & $248(52.3)$ & $238(50.2)$ & \\
\hline & Part time, n (\%) & $36(7.6)$ & $200(7.7)$ & & $36(7.6)$ & $45(9.5)$ & \\
\hline & Missing, n (\%) & $1(0.2)$ & $20(0.8)$ & & $1(0.2)$ & $1(0.2)$ & \\
\hline \multicolumn{8}{|l|}{ Maternal health status } \\
\hline Height & $\mathrm{cm}$, mean $(\mathrm{SD})$ & $160.0(5.4)$ & $159.4(5.2)$ & 0.015 & $160.0(5.4)$ & $159.8(5.1)$ & 0.75 \\
\hline Weight before pregnancy & $\mathrm{kg}$, mean (SD) & $52.4(6.9)$ & $51.5(7.8)$ & 0.011 & $52.4(6.9)$ & $52.0(7.2)$ & 0.74 \\
\hline Smoking during pregnancy & Yes, n (\%) & $6(1.3)$ & $92(3.5)$ & 0.01 & $6(1.3)$ & $6(1.3)$ & 0.27 \\
\hline Drinking during pregnancy & Yes, n (\%) & $0(0)$ & $11(0.4)$ & 0.39 & $0(0)$ & $0(0)$ & NA \\
\hline Family history of hypertension & Yes, n (\%) & $110(23.2)$ & $414(15.9)$ & $<0.001$ & $110(23.2)$ & $104(21.9)$ & 0.82 \\
\hline Systolic blood pressure $<20$ weeks of gestation & mmHg, mean (SD) & $110.4(10.4)$ & $108.4(10.2)$ & $<0.001$ & $110.4(10.4)$ & $110.9(9.9)$ & 0.75 \\
\hline Diastolic blood pressure $<20$ weeks of gestation & mmHg, mean (SD) & $64.8(7.3)$ & $62.8(7.1)$ & $<0.001$ & $64.8(7.3)$ & $65.0(7.0)$ & 0.86 \\
\hline \multicolumn{8}{|l|}{ Maternal medical complication } \\
\hline Central nervous system disease & Yes, n (\%) & $0(0)$ & $27(1.0)$ & 0.016 & $0(0)$ & $1(0.2)$ & $>0.99$ \\
\hline Cerebrovascular disease & Yes, n (\%) & $0(0)$ & $4(0.2)$ & $>0.99$ & $0(0)$ & $1(0.2)$ & $>0.99$ \\
\hline Asthma & Yes, n (\%) & $13(2.7)$ & $92(3.5)$ & 0.49 & $13(2.7)$ & $9(1.9)$ & 0.29 \\
\hline Respiratory disease (except asthma) & Yes, n (\%) & $2(0.4)$ & $3(0.1)$ & 0.17 & $2(0.4)$ & $4(0.8)$ & $>0.99$ \\
\hline Gastrointestinal disease & Yes, n (\%) & $2(0.4)$ & $33(1.3)$ & 0.15 & $2(0.4)$ & $1(0.2)$ & 0.57 \\
\hline Liver disease & Yes, n (\%) & $3(0.6)$ & $16(0.6)$ & $>0.99$ & $3(0.6)$ & $2(0.4)$ & 0.57 \\
\hline Hematologic disease & Yes, n (\%) & $2(0.4)$ & $16(0.6)$ & $>0.99$ & $2(0.4)$ & $2(0.4)$ & $>0.99$ \\
\hline Cardiovascular disease & Yes, n (\%) & $5(1.1)$ & $51(2.0)$ & 0.26 & $5(1.1)$ & $4(0.8)$ & 0.42 \\
\hline Hyperthyroidism & Yes, n (\%) & $7(1.5)$ & $47(1.8)$ & 0.85 & $7(1.5)$ & $4(0.8)$ & $>0.99$ \\
\hline Hypothyroidism & Yes, n (\%) & $13(2.7)$ & $70(2.7)$ & 0.88 & $13(2.7)$ & $14(3.0)$ & 0.29 \\
\hline Psychiatric disease & Yes, n (\%) & $10(2.1)$ & $64(2.5)$ & 0.75 & $10(2.1)$ & $9(1.9)$ & $>0.99$ \\
\hline Autoimmune disease & Yes, n (\%) & $9(1.9)$ & $55(2.1)$ & 0.86 & $9(1.9)$ & $6(1.3)$ & $>0.99$ \\
\hline Pre-existing diabetes mellitus & Yes, n (\%) & $1(0.2)$ & $15(0.6)$ & 0.49 & $1(0.2)$ & $2(0.4)$ & 0.57 \\
\hline \multicolumn{8}{|l|}{ Paternal demographics } \\
\hline Age & Year, mean (SD) & $40.8(4.8)$ & $37.3(4.9)$ & $<0.001$ & $40.8(4.8)$ & $40.6(4.8)$ & 0.25 \\
\hline Age at marriage & Year, mean (SD) & $33.3(5.3)$ & $31.7(4.8)$ & $<0.001$ & $33.3(5.3)$ & $33.2(5.6)$ & 0.37 \\
\hline
\end{tabular}


Table 1 Baseline characteristics of the eligible study sample and propensity score matched sample (Continued)

\begin{tabular}{|c|c|c|c|c|c|c|c|}
\hline \multicolumn{8}{|l|}{ Paternal health status } \\
\hline \multirow[t]{2}{*}{ Partner's smoking status } & Yes, n (\%) & $136(28.7)$ & 705 (27.0) & 0.31 & $136(28.7)$ & $140(29.5)$ & 0.84 \\
\hline & Missing, n (\%) & $6(1.3)$ & $59(2.3)$ & & $6(1.3)$ & $6(1.3)$ & \\
\hline Partner's height & cm, mean (SD) & $172.7(5.8)$ & $173.1(5.7)$ & 0.16 & $172.7(5.8)$ & $173.4(5.6)$ & 0.10 \\
\hline Partner's weight & kg, mean (SD) & $69.6(9.2)$ & $69.8(9.6)$ & 0.70 & $69.6(9.2)$ & $70.1(9.0)$ & 0.64 \\
\hline
\end{tabular}

IVF, in vitro fertilization; SD, standard deviation.

Chi-squared or Fisher's exact tests for categorical variables and t-tests for continuous variables were used for the whole study population. Conditional logistic regression for categorical variables and regression analysis using the fixed effect model for continuous variables were used in the propensity score matched sample.

Values in bold are significant at the $p=0.05$ level.

of gestation) and late- (34-38 weeks of gestation) term, and birth outcomes in the total eligible study sample $(n=3084)$ and the propensity score matched sample $(n=948)$ were compared. For comparison, chi-squared or Fisher's exact tests for categorical variables and t-tests for continuous variables were used for the whole study population, and conditional logistic regression for categorical variables and fixed effect regression for continuous variables were used in the propensity score matched sample.

To assess the association between IVF and preeclampsia, we performed a logistic regression analysis in the initial eligible study sample $(n=3084)$, unadjusted and adjusted for baseline characteristics, and performed a conditional logistic regression analysis for the propensity score matched sample $(n=948)$. All analyses were conducted using STATA MP software (version 12.0; Stata Corporation, College Station, TX).

\section{Results}

A comparison of baseline characteristics and pregnancy outcomes between the IVF $(n=474)$ and non-IVF $(n=$ $2,610)$ groups is shown in Table 1 . Women in the IVF group were older than those in the non-IVF group at the time of first visit (38.3 vs. 34.8 years, $\mathrm{p}<0.001)$ and at marriage (30.8 vs. 29.3 years, $\mathrm{p}<0.001$ ). Women in the IVF group were more likely to be nulliparous (78.1 vs. $50.5 \%, \mathrm{p}<0.001)$ and to have had abortions than those in the non-IVF group (38.6 vs. $32.3 \%, \mathrm{p}=0.009$ ). Women in the IVF group were slightly taller and weighed more than those in the non-IVF group (height, 160.0 vs. $159.4 \mathrm{~cm}, \mathrm{p}=0.015$; weight, 52.4 vs. $51.5 \mathrm{~kg}, \mathrm{p}=0.011$ ). Furthermore, women in the IVF group were less likely to smoke (1.3 vs. $3.5 \%, \mathrm{p}=0.01$ ) but more likely to have a family history of hypertension (23.2 vs. $15.9 \%, \mathrm{p}<0.001)$. Mean BP before 20 weeks of gestation, which is considered a proxy of baseline BP, was higher in the IVF group than in the non-IVF group for both systolic (110.4 vs. $108.4 \mathrm{mmHg}$, $\mathrm{p}<0.001)$ and diastolic $(64.8$ vs. $62.8 \mathrm{mmHg}, \mathrm{p}<0.001) \mathrm{BP}$. The prevalence of underlying disease was not significantly different between the IVF and non-IVF groups, except for central nervous system disease. Partners of women in the IVF group were older than those of women in the non-IVF group at the time of first visit
( 40.8 vs. 37.3 years, $\mathrm{p}<0.001)$ and at marriage $(33.5$ vs. 31.7 years, $\mathrm{p}<0.001)$.

Baseline characteristics of the IVF and non-IVF groups after propensity score matching (each $n=474$ ) were not significantly different. For example, maternal age for the IVF and non-IVF groups was 38.3 and 38.4 years $(\mathrm{p}=0.97)$, respectively, and mean diastolic BP was 64.8 and $65.0 \mathrm{mmHg}(\mathrm{p}=0.86)$ for the IVF and non-IVF groups, respectively.

Table 2 shows the characteristics of preeclampsia and BP during mid- and late-pregnancy in the IVF $(n=474)$ and non-IVF $(\mathrm{n}=2,610)$ groups. Preeclampsia was found in $15(3.2 \%)$ cases in the IVF group and $31(1.2 \%)$ cases in the non-IVF group. Among those who had preeclampsia, the number of early onset $(<32$ weeks of gestation) cases was two (13.3\%) for the IVF group and three $(9.7 \%)$ for the non-IVF group, which was not significantly different $(p=0.71)$. Further, the severity of preeclampsia was not different between the IVF and nonIVF groups: severe preeclampsia was found in eight (53.3\%) cases in the IVF group and 17 (54.8\%) cases in the non-IVF group $(\mathrm{p}=0.92)$. Mean diastolic BP during mid- and late-pregnancy was significantly higher in the IVF group than in the non-IVF group (mid-pregnancy, 64.5 vs. $62.9 \mathrm{mmHg}, \mathrm{p}<0.001$; late-pregnancy, 67.4 vs. $65.9 \mathrm{mmHg}, \mathrm{p}<0.001$ ), while systolic BP during midand late-pregnancy were not significantly different. Birth weight was higher in the IVF group than in the non-IVF group (3042.7 vs. $2988.1 \mathrm{~g}, \mathrm{p}=0.008$ ), while gestational age was not significantly different.

Table 2 also shows the characteristics of preeclampsia and BP during mid- and late-pregnancy in the propensity score matched sample (each $n=474$ ). Although the prevalence of preeclampsia in the IVF group was higher than in the non-IVF group (3.2\% vs. $1.5 \%$ ), the difference was not significant after propensity matching $(\mathrm{p}=0.27)$. Further, mean diastolic BP during mid- and late-pregnancy was not significantly different (mid-pregnancy, $64.5 \mathrm{vs} .64 .3 \mathrm{mmHg}$, $\mathrm{p}=0.99$; late-pregnancy, 67.4 vs. $67.1 \mathrm{mmHg}, \mathrm{p}=0.69$ ). Birth weight also did not significantly differ.

Table 3 shows the odds ratios (ORs) of IVF for the risk of preeclampsia in the total eligible study sample $(\mathrm{n}=$ $3,084)$, unadjusted and adjusted for baseline characteristics, 
Table 2 Characteristics of preeclampsia and blood pressure during mid- and late-pregnancy

\begin{tabular}{|c|c|c|c|c|c|c|c|}
\hline & & \multicolumn{3}{|c|}{ Eligible study sample $(n=3084)$} & \multicolumn{3}{|c|}{$\begin{array}{l}\text { Propensity score matched sample } \\
\qquad(\mathrm{n}=948)\end{array}$} \\
\hline & & $\begin{array}{l}\text { IVF group } \\
(\mathrm{n}=474)\end{array}$ & $\begin{array}{l}\text { Non-IVF group } \\
(n=2610)\end{array}$ & $P$ value & $\begin{array}{l}\text { IVF group } \\
(\mathrm{n}=474)\end{array}$ & $\begin{array}{l}\text { Non-IVF group } \\
(n=474)\end{array}$ & $P$ value \\
\hline \multicolumn{8}{|l|}{ Preeclampsia ${ }^{a}$} \\
\hline Prevalence & n (\%) & $15(3.2)$ & $31(1.2)$ & 0.003 & $15(3.2)$ & $7(1.5)$ & 0.27 \\
\hline \multicolumn{8}{|l|}{ Timing (among those with preeclampsia) } \\
\hline Early onset ( $<32$ weeks of gestation) & n (\%) & $2(13.3)$ & $3(9.7)$ & 0.71 & $2(13.3)$ & $0(0)$ & 0.27 \\
\hline Late onset ( $\geq 32$ weeks of gestation) & n (\%) & $13(86.7)$ & $28(90.3)$ & & $13(86.7)$ & $7(100)$ & \\
\hline \multicolumn{8}{|c|}{ Severity (among those with preeclampsia) } \\
\hline Mild & n (\%) & $7(46.7)$ & $14(45.2)$ & 0.92 & $7(46.7)$ & $3(42.9)$ & 0.27 \\
\hline Severe & n (\%) & $8(53.3)$ & $17(54.8)$ & & $8(53.3)$ & $4(57.1)$ & \\
\hline \multicolumn{8}{|l|}{ Blood pressure } \\
\hline SBP during mid-pregnancy ${ }^{\mathrm{b}}$ & mmHg, mean (SD) & $109.0(9.6)$ & $108.2(9.7)$ & 0.10 & $109.0(9.6)$ & $109.3(10.1)$ & 0.42 \\
\hline DBP during mid-pregnancy ${ }^{b}$ & mmHg, mean (SD) & $64.5(6.6)$ & $62.9(6.9)$ & $<0.001$ & $64.5(6.6)$ & $64.3(7.3)$ & 0.99 \\
\hline SBP during late-pregnancy ${ }^{c}$ & mmHg, mean (SD) & $112.1(9.6)$ & $111.2(9.7)$ & 0.068 & $112.1(9.6)$ & $112.1(9.0)$ & 0.35 \\
\hline DBP during late-pregnancy ${ }^{c}$ & mmHg, mean (SD) & $67.4(6.7)$ & $65.9(7.0)$ & $<0.001$ & $67.4(6.7)$ & $67.1(6.8)$ & 0.69 \\
\hline \multicolumn{8}{|l|}{ Birth outcomes } \\
\hline Birth weight & g, mean (SD) & $3042.7(425.7)$ & $2988.1(408.0)$ & 0.008 & $3042.7(425.7)$ & $3007.0(414.0)$ & 0.21 \\
\hline Gestational age & weeks, mean (SD) & $39.2(1.6)$ & $39.1(1.6)$ & 0.15 & $39.2(1.6)$ & $39.2(1.7)$ & 0.77 \\
\hline
\end{tabular}


patient is on bed rest, or if proteinuria is $\geq 2 \mathrm{~g} / 24$ hours or $\geq 3$ on dipstick in two random urine samples collected at least four hours apart.

${ }^{b}$ mid-pregnancy: 28-32 weeks of gestation.

Tate-pregnancy: 34-38 weeks of gestation.

IVF, in vitro fertilization; SD, standard deviation; SBP, systolic blood pressure; DBP, diastolic blood pressure.

Chi-squared or Fisher's exact tests for categorical variables and t-tests for continuous variables were used for the whole study population. Conditional logistic regression for categorical variables and regression analysis using the fixed effect model for continuous variables were used in the propensity score matched sample.

Values in bold are significant at the $p=0.05$ level.

and propensity score matched sample (each $n=474$ ). In the unadjusted model using the eligible study sample, IVF was a significant risk factor for preeclampsia (OR: 2.72, 95\% confidence interval $[\mathrm{CI}]: 1.46-5.08, \mathrm{p}=0.002)$. After adjusting for baseline maternal and paternal characteristics, IVF was still significantly associated with preeclampsia (OR: 2.32; 95\% CI: $1.08-4.99, \mathrm{p}=0.031$ ). However, in the propensity score matched sample, IVF was not significantly associated with preeclampsia, although the point estimation of the risk of IVF for preeclampsia was in the same positive direction as with the previous models (OR: 2.50, 95\% CI: 0.49-12.89, $\mathrm{p}=0.273$ ).

\section{Discussion}

In the present study, we used propensity score matching analysis and adjusted for 27 known variables to simulate random assignment of the IVF procedure. Using this analysis, we found that women who conceived by IVF were not at a significantly higher risk of preeclampsia than women who conceived without IVF, although the point estimation of the risk of IVF for preeclampsia was positive (i.e. 2.50), suggesting that IVF may be weakly associated with preeclampsia.

To the best of our knowledge, this is the first study to investigate the impact of IVF on preeclampsia using

Table 3 Odds ratio of developing preeclampsia among patients who had IVF

\begin{tabular}{|c|c|c|}
\hline Model & Odds ratio $(95 \% \mathrm{Cl})$ & $P$ value \\
\hline Eligible study sample, unadjusted $(n=3084)$ & $2.72(1.46-5.08)$ & 0.002 \\
\hline Eligible study sample, adjusted for baseline characteristics ${ }^{a}(n=3084)$ & $2.32(1.08-4.99)$ & 0.031 \\
\hline Propensity score matched sample (pair of $n=474$ ) & $2.50(0.49-12.89)$ & 0.273 \\
\hline
\end{tabular}

adjusted confounders were maternal characteristics (age, age at marriage, parity, number of abortions, working status, height, weight before pregnancy, smoking and drinking during pregnancy, family history of hypertension, mean systolic and diastolic blood pressure before 20-weeks gestational age, and underlying diseases such as central nervous system disease, cerebrovascular disease, asthma, hyperthyroidism, hypothyroidism, psychiatric disease, and autoimmune disease) and paternal characteristics (age, age at marriage, height, weight, and smoking status).

Multiple logistic regression was used for analysis.

Values in bold are significant at the $p=0.05$ level. 
propensity score matching analysis. In a 2004 meta-analysis of eight studies, Jackson et al. concluded that IVF was associated with an increased risk of preeclampsia (OR: 1.55; 95\% CI: 1.23-1.95) [4], although the point estimate of OR was lower than that of our study, which might be due to ethnic differences of the sample, or by chance. Moreover, the studies used in the meta-analysis adjusted for only a limited number of confounders, such as age and parity, thereby limiting the ability to adjust for assignment bias in the estimated effect. After this meta-analysis, several investigators demonstrated associations between IVF and preeclampsia [5,6,11]. Shevell et al. adjusted for more confounders (age, race, marital status, years of education, prior preterm delivery with anomaly, body mass index [BMI], smoking history, and bleeding during the current pregnancy), and reported a significant association between IVF and preeclampsia (OR: 2.7, 95\% CI: 1.7-4.4) [5]. Our study, which used propensity score matching with 27 variables, also showed a marginal impact of IVF on preeclampsia, which is an important contribution to the literature.

Specifically, we demonstrated that IVF was not significantly associated with preeclampsia, although the point estimate of OR was 2.50, which implies that IVF is a risk factor for preeclampsia. The lack of a significant association might be due to the small sample size $(n=948)$; however, attenuation of the point estimate of OR from the unadjusted model (i.e., 2.72) to the propensity score analysis (2.50) was substantial, suggesting that the association observed between IVF and preeclampsia in previous studies might have been due to confounders.

Further, the timing of onset of preeclampsia, severity of preeclampsia, and BP during mid- and late-pregnancy did not differ between the IVF and non-IVF groups. These results suggest that IVF may not elevate BP or induce preeclampsia because women with preeclampsia have higher BP levels throughout pregnancy [12]. Macdonald-Wallis et al. reported that age, BMI, and parity affected BP throughout pregnancy [13], but no single risk factor elevated BP during only early pregnancy.

A plausible biological mechanism to explain how there could be a causal relationship between IVF and preeclampsia is unclear. Abnormal placentation is considered the primary stage of pathogenesis in preeclampsia [14]. In a recent systematic review, several biological mechanisms by which IVF may be associated with preeclampsia by abnormal placentation have been proposed [7]. Transfer of the conceptus into the uterine cavity and the effect of an altered hormonal environment in the uterine myometrium during the IVF procedure may interfere with the development of the maternal-fetal interface. Additionally, as the formation of the chorion is initiated in vitro in IVF pregnancies, the inherent difference in the nature of the placenta may lead to abnormal placentation and diseased placental vessels. Furthermore, inadequate utero-placental circulation may contribute to the association between IVF and preeclampsia.

There are several limitations to our study. First, given our observational study design, we could not completely avoid selection biases and unknown confounding factors (e.g., personality and genetic traits) that existed before IVF treatment. These factors may have affected pregnancy outcomes, including the incidence of preeclampsia, despite adjusting for 27 variables using a propensity score. Moreover, as we used secondary data, assessment of subfertility was not possible, although it was reported to be associated with an increased risk of preeclampsia $[15,16]$. To overcome this limitation, a randomized controlled trial would be ideal, but not feasible. Future studies will need to investigate additional variables in order to better estimate the propensity score for undergoing IVF. Second, our study was performed at a single tertiary perinatal center, although the IVF patients were referred to the center from other fertility clinics. Finally, our study only included pregnant Japanese women. As such, we could not assess the effect of race on the association between IVF and preeclampsia.

In conclusion, we found that IVF was weakly associated with preeclampsia when using propensity score matching analysis (which provides a better estimate of causality than adjusting for confounders) than when using simple adjustments in multivariate logistic regression analysis. This suggests that the influence of the IVF procedure itself was weaker than observed in previous studies. Future studies with a larger study population that can assess more confounding variables are needed to replicate our findings.

\section{Conclusions}

Propensity score matching analysis revealed that the association between IVF and preeclampsia became weaker than with conventional adjustments in a multivariate logistic regression, suggesting that the association between IVF and preeclampsia might be confounded by residual unmeasured factors.

\section{Abbreviations \\ IVF: In vitro fertilization; BP: Blood pressure; OR: Odds ratios; Cl: Confidence interval.}

\section{Competing interests}

The authors report no conflicts of interest. The authors alone are responsible for the content and writing of the paper.

\section{Authors' contributions}

WN and FT conceived and designed the study. WN, ST, TK, YY, KK and SH contributed to clinical data collection. WN and FT contributed to statistical analysis and interpretation of data. WN drafted the manuscript and TF finalized it. All authors contributed to critical revision and final approval of the manuscript.

\section{Acknowledgements}

This study was supported by a grant from the National Center for Child Health and Development (A21-11). 


\section{Author details}

'Department of Maternal-Fetal Neonatal Medicine, National Center for Child Health and Development, 2-10-1 Okura, Setagaya, Tokyo 157-8535, Japan. ${ }^{2}$ Department of Social Medicine, National Research Institute for Child Health and Development, 2-10-1 Okura, Setagaya, Tokyo 157-8535, Japan. ${ }^{3}$ Department of Information Technology and Management, National Center for Child Health and Development, 2-10-1 Okura, Setagaya, Tokyo 157-8535, Japan.

Received: 22 February 2013 Accepted: 10 February 2014

Published: 13 February 2014

\section{References}

1. Raymond D, Peterson E: A critical review of early-onset and late-onset preeclampsia. Obstet Gynecol Surv 2011, 66(8):497-506.

2. Sibai B, Dekker G, Kupferminc M: Pre-eclampsia. Lancet 2005, 365(9461):785-799

3. Steegers EA, von Dadelszen P, Duvekot JJ, Pijnenborg R: Pre-eclampsia. Lancet 2010, 376(9741):631-644.

4. Jackson RA, Gibson KA, Wu YW, Croughan MS: Perinatal outcomes in singletons following in vitro fertilization: a meta-analysis. Obstet Gynecol 2004, 103(3):551-563.

5. Shevell T, Malone FD, Vidaver J, Porter TF, Luthy DA, Comstock CH, Hankins GD, Eddleman K, Dolan S, Dugoff L, et al: Assisted reproductive technology and pregnancy outcome. Obstet Gynecol 2005, 106(5 Pt 1):1039-1045.

6. Chen XK, Wen SW, Bottomley J, Smith GN, Leader A, Walker MC: In vitro fertilization is associated with an increased risk for preeclampsia. Hypertens Pregnancy 2009, 28(1):1-12.

7. Thomopoulos C, Tsioufis C, Michalopoulou H, Makris T, Papademetriou V, Stefanadis C: Assisted reproductive technology and pregnancy-related hypertensive complications: a systematic review. J Hum Hypertens 2013, 27(3):148-157.

8. Joffe MM, Rosenbaum PR: Invited commentary: propensity scores. Am J Epidemiol 1999, 150(4):327-333.

9. Leuven E, Sianesi B. PSMATCH2: Stata module to perform full Mahalanobis and propensity score matching, common support graphing, and covariate imbalance testing. 2003. [http://ideas.repec.org/c/boc/bocode/ s432001.html].

10. Guo S, Fraser MW: Propensity Score Analysis. Thausand Oaks, CA: SAGE Publications, Inc; 2010

11. Kallen B, Finnstrom O, Lindam A, Nilsson E, Nygren KG, Otterblad Olausson $P$ : Trends in delivery and neonatal outcome after in vitro fertilization in Sweden: data for 25 years. Hum Reprod 2010, 25(4):1026-1034

12. Gaillard R, Bakker R, Willemsen SP, Hofman A, Steegers EA, Jaddoe WW: Blood pressure tracking during pregnancy and the risk of gestational hypertensive disorders: the generation R study. Eur Heart J 2011, 32(24):3088-3097.

13. Macdonald-Wallis C, Tilling K, Fraser A, Nelson SM, Lawlor DA: Established preeclampsia risk factors are related to patterns of blood pressure change in normal term pregnancy: findings from the Avon longitudinal study of parents and children. J Hypertens 2011, 29(9):1703-1711.

14. Lain KY, Roberts JM: Contemporary concepts of the pathogenesis and management of preeclampsia. JAMA 2002, 287(24):3183-3186.

15. Basso O, Weinberg CR, Baird DD, Wilcox AJ, Olsen J: Subfecundity as a correlate of preeclampsia: a study within the Danish National Birth Cohort. Am J Epidemiol 2003, 157(3):195-202.

16. Trogstad L, Magnus P, Moffett A, Stoltenberg C: The effect of recurrent miscarriage and infertility on the risk of pre-eclampsia. BJOG 2009, 116(1):108-113.

doi:10.1186/1471-2393-14-69

Cite this article as: Watanabe et al: Is in vitro fertilization associated with preeclampsia? A propensity score matched study. BMC Pregnancy and Childbirth 2014 14:69.

\section{Submit your next manuscript to BioMed Central and take full advantage of:}

- Convenient online submission

- Thorough peer review

- No space constraints or color figure charges

- Immediate publication on acceptance

- Inclusion in PubMed, CAS, Scopus and Google Scholar

- Research which is freely available for redistribution
C Biomed Central 